\title{
Household clustering of asymptomatic malaria infections in Xepon district, Savannakhet province, Lao PDR
}

Tiengkham Pongvongsa ${ }^{1,2,3}$, Daisuke Nonaka ${ }^{3,4^{*}}$, Moritoshi Iwagami ${ }^{3,5,8}$, Masami Nakatsu ${ }^{3,5}$, Panom Phongmany' ${ }^{1}$ Futoshi Nishimoto ${ }^{6}$, Jun Kobayashi, ${ }^{3,4}$, Bouasy Hongvanthon ${ }^{3,7}$, Paul T. Brey ${ }^{3,8}$, Kazuhiko Moji ${ }^{6}$, Toshihiro Mita ${ }^{2,3}$ and Shigeyuki Kano ${ }^{3,5,8}$

\begin{abstract}
Background: In the Lao PDR, malaria morbidity and mortality have remarkably decreased over the past decade. However, asymptomatic infections in rural villages contribute to the on-going local transmission. The primary objective of this study was to explore the characteristics of infections in a malaria-endemic district of the Lao PDR. The specific objectives were to investigate the prevalence and species of malaria parasites using molecular methods and to assess individual and household parasite levels and the characteristics associated with malaria infection.

Methods: The study population included 870 participants from 236 households in 10 villages of the Xepon district. Interviews, blood examinations and body temperature measurements were conducted between August and September 2013. A multilevel logistic regression model, with adjustment for clustering effects, was used to assess the association between predictor variables and an outcome variable (malaria infection status as principally determined by PCR). The predictive factors included individual-level factors (age, gender, past fever episode, and forest activity during night time) and household-level factors (household member size, household bed net usage/density and a household with one other malaria-infected member).

Results: Fifty-two participants (including 26 children) tested positive (positive rate: $6.0 \%$ ): Plasmodium falciparum mono-infection was the most common infection $(n=41,78.8 \%)$, followed by P. falciparum and Plasmodium vivax mixed infections $(n=9,17.3 \%)$. The majority of infected participants $(n=42,80.8 \%)$ had no fever episodes in the two previous weeks or a measurable fever $\left(>37^{\circ} \mathrm{C}\right)$ at the time of survey. Living in a household with one other malaria-infected member significantly increased the odds of infection (odds ratio $24.33,95 \%$ confidence interval 10.15-58.32). Among the 40 households that had at least one infected member, nine households were responsible for $40.4 \%$ of the total infections.

Conclusions: Plasmodium vivax was detected more frequently than it was reported from the district hospital. Most infections were asymptomatic and sub-microscopic and were highly clustered within households. To further eliminate malaria in Xepon and other similar settings in the country, the National Malaria Control Programme should consider household-based strategies, including reactive case detection targeting the household members of index cases.
\end{abstract}

Keywords: Asymptomatic infections, Sub-microscopic infections, Malaria elimination, Active case detection, Laos

\footnotetext{
*Correspondence: laodaisuke@hotmail.co.jp

${ }^{4}$ Department of Global Health, School of Health Sciences, University of the Ryukyus, 207 Uehara, Nishihara-cho, Okinawa 903-0215, Japan Full list of author information is available at the end of the article
} 


\section{Background}

The Lao People's Democratic Republic (Lao PDR) is a lower-middle-income country in Southeast Asia, bordering Thailand, Vietnam, Cambodia, China, and Myanmar. The population of the Lao PDR was 6.5 million in 2015, and $63 \%$ of the population lives in rural areas [1]. The predominant crop is rice, and $71 \%$ of the households in the nation were engaged in rice farming in 2010/11 [2]. Two-thirds of the land is either hilly or mountainous. The Lao PDR has a monsoonal climate. The rainy season extends from April to November, with peak rainfall in July and August [1,2]. Although malaria cases are reported from healthcare facilities throughout the year, there is an increase in the number of reported cases during the rainy season [3]. Plasmodium falciparum has long been the predominant reported infection, but the incidence Plasmodium vivax has been increasing in recent years [4]. The groups most at risk of malaria infection in the Lao PDR include ethnic minorities, forest fringe inhabitants, migrant workers, and new forest settlers $[3,5,6]$. People in these groups live or work in the forest where they are engaged in cropping, hunting, collecting of forest products, mining, or constructing roads/ dams. Therefore, their exposure to vector mosquitoes is increased. Among the ethnic minorities and forest fringe inhabitants, adult males in particular report that they stay in the forest overnight to hunt or collect wood $[3,7$, 8].

Since 1992, the Lao PDR has implemented a nationwide malaria control programme [8]. The current malaria control strategies emphasize the promotion of longlasting insecticide-treated bed nets, early diagnosis by microscopic examination and rapid diagnostic tests (RDT), and prompt treatment with an artemisinin-based combination therapy (ACT). Since 2008, the use of ACT has gradually been scaled-up to cover the whole public health sector, including village health volunteers (community health workers) and some businesses in the private sector, including registered private pharmacies [3].

As a result of the above-mentioned control efforts, there have been remarkable decreases in the rates of mortality and morbidity due to malaria over the past decade. The Lao PDR achieved a $>75 \%$ decrease in the incidence of malaria between 2000 and 2015. However, asymptomatic infections continue to be detected in rural, remote villages $[7,9,10]$, which contributes to on-going local transmission. Asymptomatic carriers are not detected by the current national surveillance system, which is based on passive case detection at healthcare facilities. Furthermore, patients who are symptomatic may not necessarily seek treatment from healthcare facilities in which the surveillance system is in place [11]. Thus, the actual intensity of malaria transmission in the communities of the Lao PDR remains poorly understood.

Artemisinin resistance has been increasingly reported from many areas in the Greater Mekong Sub-region (Lao PDR, Cambodia, Myanmar, Thailand, Vietnam, and China) [12], posing a threat to regional and global health security. In 2014, the Malaria Policy Advisory Committee to the World Health Organization reviewed the situation of the region, considered malaria elimination as technically and operationally feasible, and thus recommended that the regional countries adopt a goal of malaria elimination by 2030 [13]. The elimination strategies in each country should be based on the local epidemiology [13]. However, information is lacking to guide and implement elimination strategies in the Lao PDR. A communitybased survey with a highly sensitive molecular measurement method [i.e., polymerase chain reaction (PCR)] can help to better understand the epidemiological trends in the communities of the Lao PDR.

Approximately $90 \%$ of all malaria cases in the Lao PDR occur in the southernmost provinces including Savannakhet province [3]. Of the 15 districts in this province, malaria is endemic in four districts including Xepon district. Xepon district is a remote, rural district on the Vietnamese border, approximately $500 \mathrm{~km}$ from the national capital of Vientiane. Ethnic minorities comprise $75 \%$ of the total district population, which is approximately 45,000 . There are 88 villages in the district, most of which are at risk of malaria. According to the Xepon District Health Office, 163 malaria cases were reported from the Xepon District Hospital in 2013, including 151 cases of $P$. falciparum mono-infection, 10 cases of $P$. vivax monoinfection, and two cases of co-infections. In 2012, 218 cases were reported, including 214 cases of $P$. falciparum mono-infection and four cases of $P$. vivax mono-infection. Xepon district was deliberately selected because reliable demographic data were available on households in the 20 villages in which the Health and Demographic Surveillance System (HDSS) was in place. The HDSS has been described elsewhere [14].

The primary objective of this study was to explore the characteristics of individuals with malaria infections in a rural district of the Lao PDR. The specific objectives were (1) to investigate the prevalence and species of malaria parasites in the study community using molecular methods, and (2) to assess the individual- and household-level characteristics that are associated with malaria infection.

\section{Methods}

\section{Study site and population}

This study was conducted in Xepon district, Savannakhet province, Lao PDR between August and September 
of 2013. From the 20 villages where the HDSS was in place, 10 villages were deliberately selected as the study site based on the geographical characteristics of these villages: two hillside villages, five roadside villages, and three riverside villages were selected (Fig. 1). Data were collected from 870 participants in 236 households (participation rate: $47.8 \%$ ).

\section{Data collection}

Data were collected through a cross-sectional, community-based survey. The survey consisted of interviews, blood examinations, and body temperature measurements. All of the villagers in each study village were invited to assemble at a designated place, including a primary school and village health volunteers' houses. First, trained surveyors, who were nurses or medical technologists, identified a participant by his/her name, the name of his/her household head, and the household ID used for the HDSS. Second, surveyors conducted an interview using a pre-tested questionnaire. In the case of child participants, a guardian responded to the interview. Third, the surveyors measured the body temperature of the participants with a digital ear thermometer (MC510, Omron Healthcare Co., Ltd., Kyoto, Japan). Finally, the surveyors collected a finger-prick blood sample from the participants that was subjected to RDT (SD Bioline
Malaria Ag Pf/Pv, Standard Diagnostics, Inc., Gyeonggido, Republic of Korea). Blood samples were also collected for microscopic examination and PCR. For PCR, blood samples were collected on Whatman FTA Classic Cards (GE Healthcare Life Science, Little Chalfont, UK).

\section{Laboratory procedures \\ Microscopy}

Experienced medical technologists prepared Giemsastained thick and thin blood smears and performed the microscopic examinations.

\section{DNA extraction and $P C R$}

Deoxyribonucleic acid (DNA) was extracted from dried blood spots on the FTA Classic Cards with a QIAamp DNA Mini Kit (Qiagen, Hilden, Germany) in accordance with the manufacturer's instructions. The extracted DNA was eluted with $50 \mu \mathrm{l}$ of elution buffer in the kit and preserved at $4{ }^{\circ} \mathrm{C}$ until the PCR. The PCR was performed in two steps. First, screening by a real-time PCR was performed with genus-specific primers targeting the cytochrome $b$ gene in the mitochondrial genome of Plasmodium parasites [15]. Second, Plasmodium species identification was performed with species-specific primer sets targeting the same gene by a nested PCR with DNA samples that were identified as being Plasmodium

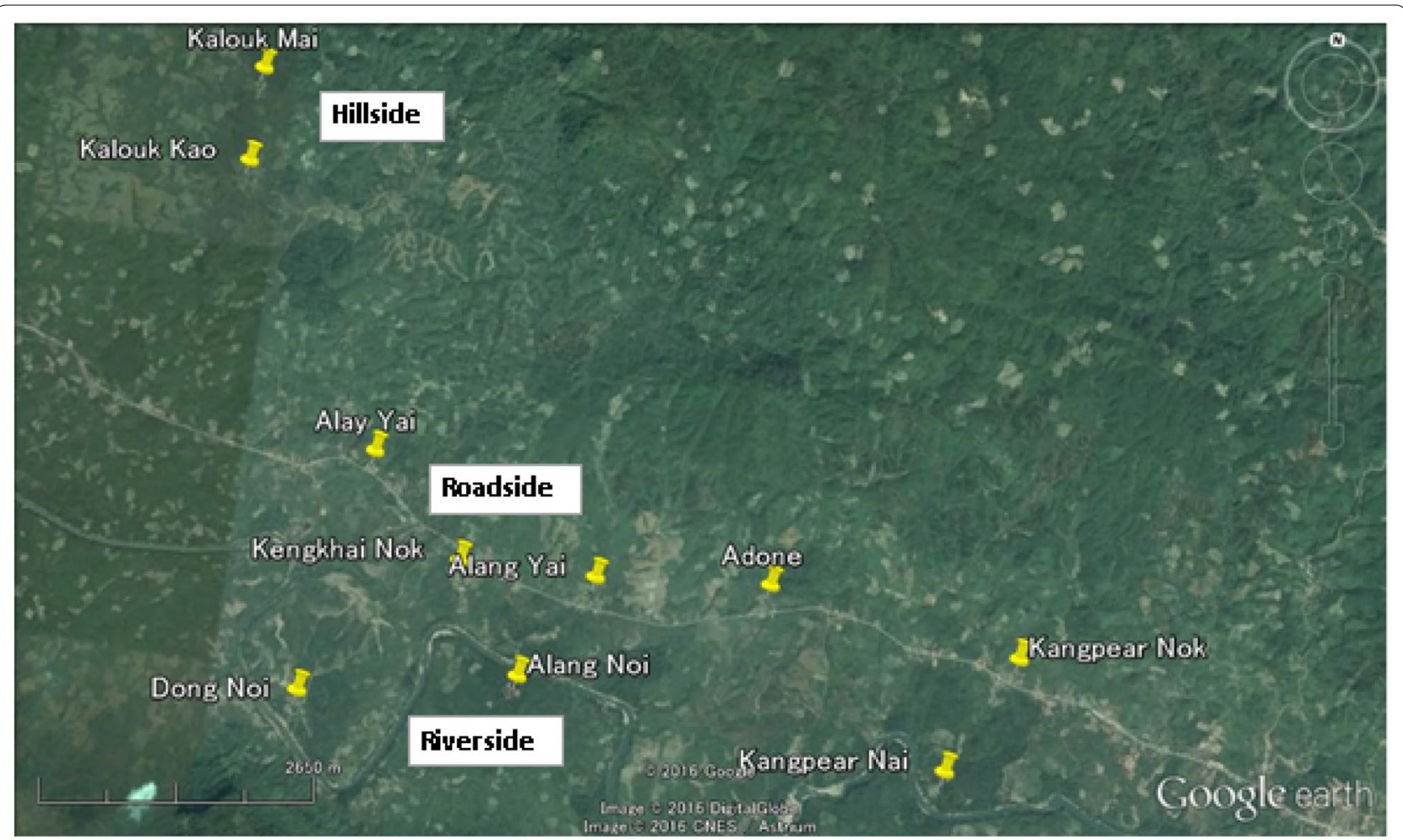

Fig. 1 Map of the study villages. Yellow pins indicate the locations of the study villages 
parasite-positive in the screening. SaoAdvanced Universal SYBR Green Supermix (Bio-Rad, CA, USA) was used for the screening PCR with $2 \mu \mathrm{l}$ of the extracted DNA and the primer set (Additional file 1). When the melting temperature of the PCR product was between 76.5 and $78.5^{\circ} \mathrm{C}$, the sample was considered to be Plasmodium DNA positive.

For the PCR for species identification, primer sets for P. falciparum, P. vivax, Plasmodium malariae, and Plasmodium ovale were used. The primary PCR for the identification was conducted by the real-time PCR. The secondary PCR for the identification was conducted using a conventional PCR with the first PCR product diluted 1000 times with Go-Taq DNA Polymerase (Promega, Fitchburg, WI, USA). The PCR products were visualized by $2 \%$ agarose gel electrophoresis with Gel Red (Biotium, Fremont, CA, USA) staining by UV transillumination. All of the DNA extraction procedures and PCRs were conducted at the National Center for Global Health and Medicine, Japan.

\section{Factors and measurements}

The primary outcome measure in this study was the malaria infection status principally determined by PCR. The secondary outcome measure was the malaria infection status determined by RDT and then confirmed principally by PCR, i.e., true positives detected by RDT. Malaria infection was defined by a positive test result with or without clinical symptoms. The individual-level predictive factors included age, gender, bed net use on the night before the survey, the experience of working in the forest at night during the previous month, and the experience of a fever during the two weeks prior to the survey. The age and gender variables were determined from the information in the HDSS database because the self-reported ages of the subjects at the study site might not have been reliable. Other variables were measured using a questionnaire-based interview. At the household level, the predictive factors included the number of household members, the person-per-net ratio (defined as the number of household members divided by the number of available bed nets in a household), and living in a household with one other malaria-infected member. These household-level factors were determined according to the information in the HDSS database and the results of a bed net observation survey that was conducted prior to the present study [16].

\section{Statistical analysis}

For the analysis of associations between the outcomes and predictive factors, multi-level modelling was used to account for the hierarchical structure of the data; i.e., 870 individuals in 236 households. Thus, a two-level, mixed-effects logistic regression model was used for the multivariate analysis. For the bi-variate analysis, a Chi square test or Fisher's exact test was used. For other statistical analyses, a Chi square test or Fisher's exact test was used for a categorical variable and a t test was used for a discrete variable. A $p<0.05$ was considered statistically significant. All statistical analyses were performed with the STATA MP12 software program (StataCorp LP, College Station, TX, USA).

\section{Results}

\section{Infection characteristics}

Fifty-two participants were confirmed to have malaria parasitaemia: 49 individuals were confirmed by PCR and the other three individuals, whose filter paper samples were not available, were determined by microscopic examination (Table 1). The total positive rate was $6.0 \%$ (52/870). Mono-infection with P. falciparum was the most common form of infection $(\mathrm{n}=41,78.8 \%)$, followed by mixed infection with $P$. falciparum and $P$. vivax ( $\mathrm{n}=9,17.3 \%$ ). RDT and microscopic examinations could only detect $36.7 \%$ of the PCR-diagnosed infections.

\section{Characteristics of individuals}

Approximately one half of the participants $(54.0 \%)$ were female (Table 2), and nearly one half of the participants

\section{Table 1 Blood examination results}

\begin{tabular}{|c|c|c|}
\hline \multicolumn{3}{|l|}{ Species } \\
\hline \multicolumn{3}{|l|}{ Total $(n=52)$} \\
\hline P. falciparum mono-infection & 41 & 78.8 \\
\hline P. vivax mono-infection & 2 & 3.8 \\
\hline P. falciparum and P. vivax mixed infection & 9 & 17.3 \\
\hline \multicolumn{3}{|l|}{ PCR positives $(n=49)$} \\
\hline P. falciparum mono-infection & 38 & 77.6 \\
\hline P. vivax mono-infection & 2 & 4.1 \\
\hline P. falciparum and P. vivax mixed infection & 9 & 18.4 \\
\hline \multicolumn{3}{|l|}{ Rapid diagnostic test positives $(\mathrm{n}=21$ ) } \\
\hline P. falciparum mono-infection & 19 & 90.5 \\
\hline P. vivax mono-infection & 1 & 4.8 \\
\hline P. falciparum and P. vivax mixed infection & 1 & 4.8 \\
\hline \multicolumn{3}{|l|}{ Microscopic examination positives $(n=21)$} \\
\hline P. falciparum mono-infection & 18 & 85.7 \\
\hline P. vivax mono-infection & 3 & 14.3 \\
\hline P. falciparum and P. vivax mixed infection & 0 & 0.0 \\
\hline \multicolumn{3}{|c|}{ Sensitivity of rapid diagnostic test against PCR positives } \\
\hline Positive & 18 & 36.7 \\
\hline Negative & 31 & 63.3 \\
\hline \multicolumn{3}{|c|}{ Sensitivity of microscopic examination against PCR positives } \\
\hline Positive & 18 & 36.7 \\
\hline Negative & 31 & 63.3 \\
\hline
\end{tabular}


Table 2 Characteristics of the participants

\begin{tabular}{|c|c|c|c|c|c|c|}
\hline \multirow[t]{2}{*}{ Characteristics } & \multicolumn{2}{|c|}{ Total $(n=870)$} & \multicolumn{2}{|c|}{ Positive $(n=52)$} & \multicolumn{2}{|c|}{ Negative $(n=818)$} \\
\hline & $\mathbf{n}$ & $\%$ & $\mathbf{n}$ & $\%$ & $\mathbf{n}$ & $\%$ \\
\hline \multicolumn{7}{|l|}{ Gender } \\
\hline Female & 470 & 54.0 & 24 & 47.1 & 446 & 54.5 \\
\hline Male & 400 & 46.0 & 27 & 52.9 & 373 & 45.5 \\
\hline \multicolumn{7}{|l|}{ Age (years) } \\
\hline$<5$ & 67 & 7.7 & 1 & 1.9 & 66 & 8.1 \\
\hline $5-14$ & 392 & 45.1 & 25 & 48.1 & 367 & 44.9 \\
\hline$\geq 15$ & 411 & 47.2 & 26 & 50.0 & 385 & 47.1 \\
\hline \multicolumn{7}{|c|}{ Slept under a bed net last night } \\
\hline Yes & 863 & 99.2 & 52 & 100.0 & 811 & 99.1 \\
\hline No & 7 & 0.8 & 0 & 0.0 & 7 & 0.9 \\
\hline \multicolumn{7}{|c|}{ Worked in forest at night in the past month } \\
\hline Yes & 33 & 3.8 & 2 & 3.8 & 31 & 3.8 \\
\hline No & 837 & 96.2 & 50 & 96.2 & 787 & 96.2 \\
\hline \multicolumn{7}{|c|}{ Slept in forest/hut in the past month } \\
\hline Yes & 8 & 0.9 & 1 & 1.9 & 7 & 0.9 \\
\hline No & 862 & 99.1 & 51 & 98.1 & 811 & 99.1 \\
\hline \multicolumn{7}{|c|}{ Fever episode in the past 2 weeks } \\
\hline Yes & 71 & 8.2 & 4 & 7.7 & 67 & 8.2 \\
\hline No & 799 & 91.8 & 48 & 92.3 & 751 & 91.8 \\
\hline \multicolumn{7}{|l|}{ Body temperature $\left({ }^{\circ} \mathrm{C}\right)$} \\
\hline$\leq 37$ & 682 & 77.2 & 43 & 82.7 & 629 & 76.9 \\
\hline$>37$ & 198 & 22.8 & 9 & 17.3 & 189 & 23.1 \\
\hline Mean (standard deviation) & $36.8(0.4)$ & & $36.8(0.6)$ & & $36.8(0.4)$ & \\
\hline
\end{tabular}

(47.2\%) were over 15 years of age. Bed net use was very common, and almost all of the participants (99.2 \%) reported that they slept under a bed net on the night before the survey. Most of the participants did not work in the forests at night (96.2 \%) and had not slept in forest/hut $(99.1 \%)$ over the past month. Most of the participants $(91.8 \%)$ had not experienced a fever episode in the past 2 weeks; this was also the case for the participants who showed positive test results (92.3\%). Most of the participants $(77.2 \%)$ did not have a measurable fever; this was also the case among the participants who showed positive test results $(82.7 \%)$.

The majority ( $\mathrm{n}=42,80.8 \%$ ) of the participants with positive test results did not have a fever episode in the two weeks before the survey or a measurable fever $\left(>37^{\circ} \mathrm{C}\right)$ at the time of the survey (Additional file 2).

\section{Demographic differences between participants and non-participants}

There were significant differences in gender and age between the study participants and non-participants in the study villages (Table 3 ). The proportion of males was significantly lower in the participants than in the non-participants (46.0 vs $51.8 \%, p=0.012$ ). The proportion of the population aged over 14 years of age was significantly lower in the participants than in the nonparticipants ( 47.1 vs $60.1 \%, p<0.001$ ). There was also a difference in gender proportion between the participants and non-participants in the age subgroups: among the participants, males accounted for $53.7 \%(36 / 67)$ in the youngest group, $49.7 \%$ (195/392) in the second youngest group, and $40.6 \%(167 / 411)$ in the oldest group. In contrast, among the non-participants, males accounted for $51.5 \%(102 / 198)$ in the youngest group, $45.3 \%(82 / 181)$ in the second youngest group, and $54.0 \%(309 / 572)$ in the oldest group.

\section{Household characteristics}

Most households $(89.4 \%)$ possessed at least one bed net (Table 4). According to the person-per-net ratio, $53.0 \%$ of the households were considered to have a sufficient number of bed nets to cover all members of the household. Among the households with at least one infected person, $57.5 \%$ of the households were considered to have a sufficient number of bed nets. The mean number of household members was 6.5 . 
Table 3 Differences in demographic characteristics between participants and non-participants

\begin{tabular}{|c|c|c|c|c|c|c|c|}
\hline \multirow[t]{2}{*}{ Demographic characteristics } & \multicolumn{2}{|c|}{$\begin{array}{l}\text { Total } \\
(n=1821)\end{array}$} & \multicolumn{2}{|c|}{$\begin{array}{l}\text { Participants } \\
(\mathrm{n}=870)\end{array}$} & \multicolumn{2}{|c|}{$\begin{array}{l}\text { Non-participants } \\
(n=951)\end{array}$} & \multirow[t]{2}{*}{$P$ value ${ }^{a}$} \\
\hline & $\mathbf{n}$ & $\%$ & $\mathbf{n}$ & $\%$ & $\mathrm{n}$ & $\%$ & \\
\hline \multicolumn{8}{|l|}{ Gender } \\
\hline Female & 928 & 51.0 & 470 & 54.0 & 458 & 48.2 & \multirow[t]{2}{*}{0.012} \\
\hline Male & 893 & 49.0 & 400 & 46.0 & 493 & 51.8 & \\
\hline \multicolumn{8}{|l|}{ Age (years) } \\
\hline$<5$ & 265 & 14.6 & 67 & 7.7 & 198 & 20.8 & \multirow[t]{3}{*}{$<0.001$} \\
\hline $5-14$ & 573 & 31.5 & 392 & 45.1 & 181 & 19.0 & \\
\hline$\geq 15$ & 983 & 54.0 & 411 & 47.1 & 572 & 60.1 & \\
\hline
\end{tabular}

a Chi square test

Table 4 Characteristics of the households

\begin{tabular}{|c|c|c|c|c|c|c|}
\hline \multirow[t]{2}{*}{ Characteristics } & \multicolumn{2}{|c|}{$\begin{array}{l}\text { Total } \\
(n=236)\end{array}$} & \multicolumn{2}{|c|}{$\begin{array}{l}\text { Households with at least } \\
\text { one positive participant } \\
(n=40)\end{array}$} & \multicolumn{2}{|c|}{$\begin{array}{l}\text { Households without a } \\
\text { positive participant } \\
(n=196)\end{array}$} \\
\hline & $\mathrm{n}$ & $\%$ & $\mathrm{n}$ & $\%$ & $\mathrm{n}$ & $\%$ \\
\hline \multicolumn{7}{|l|}{ Possession of bed net } \\
\hline Yes & 211 & 89.4 & 35 & 89.7 & 176 & 89.3 \\
\hline No & 4 & 1.7 & 0 & 0.0 & 4 & 2.0 \\
\hline Unknown & 21 & 8.9 & 4 & 10.3 & 17 & 8.6 \\
\hline \multicolumn{7}{|l|}{ Person-per-net ratio } \\
\hline$\leq 2.5$ & 125 & 53.0 & 23 & 57.5 & 102 & 52.0 \\
\hline$>2.5$ and $<4.0$ & 50 & 21.2 & 9 & 22.5 & 41 & 20.9 \\
\hline$\geq 4.0$ or no net & 39 & 16.5 & 4 & 10.0 & 35 & 17.9 \\
\hline Unknown & 22 & 9.3 & 4 & 10.0 & 18 & 9.2 \\
\hline \multicolumn{7}{|l|}{ Number of members } \\
\hline$<5$ & 57 & 24.2 & 8 & 20.0 & 49 & 25.0 \\
\hline $5-7$ & 104 & 44.1 & 17 & 42.5 & 87 & 44.4 \\
\hline$\geq 8$ & 75 & 31.8 & 15 & 37.5 & 60 & 30.6 \\
\hline Mean (standard deviation) & $6.5(2.8)$ & & 6.8 & & 6.4 & \\
\hline
\end{tabular}

Among the 40 households with at least one infected member, nine households with at least two infected members were responsible for $40.4 \%$ of the total infections (Additional file 3). Among these nine households, four households $(44.4 \%)$ had a sufficient number of bed nets. In contrast, among the 27 households that had only one infected member and that provided bed net data, 19 households $(70.4 \%)$ had a sufficient number of bed nets. Although there was a tendency for the households with two or more infected members to be less likely to have a sufficient number of bed nets as compared to the households with only one infected member, the difference (44.4 vs $70.4 \%$ ) was not statistically significant ( $p=0.235$ by Fisher's exact test). Among the nine households that had at least two infected members, the mean number of members (standard deviation) was 7.2 (2.6), whereas it was 6.7 (2.5) among the 31 households that had only one infected member. The difference (7.2 vs 6.7$)$ was not statistically significant ( $p=0.574$ by t test).

\section{Village characteristics}

With the exception of one village, all of the villages had at least one malaria-positive participant (Table 5). The malaria positive rate, which ranged from 0 to $20.8 \%$, differed markedly among the villages (median: $6.0 \%$ ). The highest positive rate was found in the riverside villages. However, infected individuals were also found in hillside and roadside villages. 
Table 5 Characteristics of the villages

\begin{tabular}{lccc}
\hline Village name & $\begin{array}{l}\text { Number } \\
\text { of participants }\end{array}$ & $\begin{array}{l}\text { Number of participants } \\
\text { who tested positive }\end{array}$ & $\begin{array}{l}\text { Positive } \\
\text { rate (\%) }\end{array}$ \\
\hline Hillside & & 1 & 2.2 \\
Kalouk Mai & 46 & 12 & 10.4 \\
Kalouk Kao & 115 & & \\
Roadside & & 6 & 6.1 \\
Kangkai Nok & 98 & 7 & 5.8 \\
Adone & 121 & 7 & 7.5 \\
Alang Yai & 93 & 0 & 0.0 \\
Alay Yai & 66 & 1 & 1.0 \\
Kangpear & 104 & & \\
$\quad$ Nok & & 6 & 8.6 \\
Riverside & & 11 & 1.0 \\
Kangpear Nai & 70 & 1 & \\
Alang Noi & 53 & & \\
Dong Noi & 104 & &
\end{tabular}

\section{Factors associated with malaria infection}

\section{Association with malaria infection status principally} determined by $P C R$

The bivariate analysis showed that living in a household with at least one additional infected member was significantly associated with malaria infection $(p<0.001)$ (Table 6$)$. No other variables were found to be associated with malaria infection. Consistent with the results of the bivariate analysis, the multivariate analysis showed that living in a household with at least one additional infected member was significantly associated with an increased risk of infection (adjusted odds ratio 24.33, $95 \%$ confidence interval 10.1558.32) (Table 7).

\section{Association with malaria infection status determined by rapid diagnostic tests}

The bivariate analysis showed that living in a household with at least one additional infected member was significantly associated with malaria infection $(p<0.001$ by Fisher's exact test) (Table 8 ). No other variables were found to be significantly associated with malaria infection. However, there was a tendency for the positive participants to be more likely to have reported a fever episode as compared to the negative participants (19.0 vs $7.9 \%, p=0.084$ by Fisher's exact test). Consistent with the results of the bivariate analysis, the multivariate analysis showed that living in a household with at least one additional infected member was significantly associated with an increased risk of infection (adjusted odds ratio 56.69, 95 \% confidence interval 9.38-342.61) (Table 9).
Table 6 Bivariate analysis of associations between malaria infection status principally determined by PCR and predictive factors

\begin{tabular}{|c|c|c|}
\hline Predictive factors & Positive rate (\%) & $P$ value \\
\hline \multicolumn{3}{|c|}{ Individual level factors } \\
\hline \multicolumn{3}{|l|}{ Age (years) } \\
\hline$<5$ & 1.5 & 0.273 \\
\hline $5-14$ & 6.4 & \\
\hline$\geq 15$ & 6.3 & \\
\hline \multicolumn{3}{|l|}{ Gender } \\
\hline Female & 5.1 & 0.240 \\
\hline Male & 7.0 & \\
\hline \multicolumn{3}{|c|}{ Working forest at night } \\
\hline No & 6.0 & 0.984 \\
\hline Yes & 6.1 & \\
\hline \multicolumn{3}{|c|}{ Fever episode in the last 2 weeks } \\
\hline No & 6.0 & 1.000 \\
\hline Yes & 5.6 & \\
\hline \multicolumn{3}{|c|}{ Household level factors } \\
\hline \multicolumn{3}{|l|}{ Person-per-net ratio } \\
\hline$\leq 2.5$ & 6.2 & 0.243 \\
\hline$>2.5$ and $<4.0$ & 7.2 & \\
\hline$\geq 4.0$ or no net & 3.2 & \\
\hline Unknown & 6.7 & \\
\hline \multicolumn{3}{|c|}{ Number of household members } \\
\hline$<5$ & 7.6 & 0.689 \\
\hline $5-7$ & 5.4 & \\
\hline$\geq 8$ & 6.0 & \\
\hline \multicolumn{3}{|c|}{ Household with at least one additional infected member } \\
\hline No & 3.8 & $<0.001$ \\
\hline Yes & 45.6 & \\
\hline
\end{tabular}

a Chi square test or Fisher's exact test

\section{Discussion}

As expected from the patient records of the Xepon District Hospital, the present community-based study showed that $P$. falciparum mono-infection accounted for the majority of infections (78.8\%). However, the results of the present study also showed a difference in the proportion of $P$. falciparum and $P$. vivax infections in the patient records and the present study: $P$. vivax infections including co-infections with $P$. falciparum, which were rarely reported from the hospital in $2012(1.8 \%)$ and $2013(7.9 \%)$, accounted for $21.2 \%$ of the infections that were detected in the present study. This suggests that the burden of vivax malaria is underestimated in the district.

Consistent with the findings of a previous study conducted in Savannakhet province [9], the present study found that most of the infections (63.3\%) were asymptomatic and sub-microscopic. This finding highlighted the importance 


\begin{tabular}{|c|c|c|c|}
\hline $\begin{array}{l}\text { Predictor vari- } \\
\text { ables }\end{array}$ & $\begin{array}{l}\text { Adjusted odds } \\
\text { ratio }^{\mathrm{a}}\end{array}$ & $\begin{array}{l}\text { Adjusted } 95 \% \\
\text { confidence } \\
\text { interval }\end{array}$ & $P$ value \\
\hline
\end{tabular}

\begin{tabular}{|c|c|c|c|}
\hline \multicolumn{4}{|c|}{ Individual level variables } \\
\hline \multicolumn{4}{|c|}{ Age (years) } \\
\hline$<5$ & 1.00 & Reference & \\
\hline $5-14$ & 5.17 & $0.59-45.10$ & 0.137 \\
\hline$\geq 15$ & 4.42 & $0.51-38.52$ & 0.179 \\
\hline \multicolumn{4}{|l|}{ Gender } \\
\hline Female & 1.00 & Reference & \\
\hline Male & 1.14 & $0.60-2.17$ & 0.689 \\
\hline \multicolumn{4}{|c|}{ Working forest at midnight } \\
\hline No & 1.00 & Reference & \\
\hline Yes & 1.62 & $0.33-8.09$ & 0.555 \\
\hline \multicolumn{4}{|c|}{ Fever episode in the last 2 weeks } \\
\hline No & 1.00 & Reference & \\
\hline Yes & 1.32 & $0.39-4.46$ & 0.656 \\
\hline \multicolumn{4}{|c|}{ Household level variables } \\
\hline \multicolumn{4}{|c|}{ Person-per-net ratio } \\
\hline$\leq 2.5$ & 1.00 & Reference & \\
\hline$>2.5$ and $<4.0$ & 0.62 & $0.26-1.49$ & 0.287 \\
\hline$\geq 4.0$ or no net & 0.59 & $0.19-1.83$ & 0.360 \\
\hline \multicolumn{4}{|c|}{ Number of household members } \\
\hline$<5$ & 1.00 & Reference & \\
\hline $5-7$ & 0.67 & $0.27-1.68$ & 0.390 \\
\hline$\geq 8$ & 0.68 & $0.24-1.92$ & 0.469 \\
\hline \multicolumn{4}{|c|}{ Household with at least one additional infected member } \\
\hline No & 1.00 & Reference & \\
\hline Yes & 24.33 & $10.15-58.32$ & $<0.001$ \\
\hline
\end{tabular}

a Adjusted for age, gender, working in forest, fever episode, person per net ratio, number of household members, and at least one additional infected member

of tackling asymptomatic infections or "hidden malaria" in malaria elimination efforts in the malaria-prone districts of the Lao PDR. Thus far, no specific strategy for identifying asymptomatic parasite carriers has been implemented in the Lao PDR. Therefore, the introduction of active case detection for asymptomatic infections is recommended.

The results of the present study indicated that infections were highly clustered at the household level and that infections were unevenly distributed across households. A considerable number of studies have reported household clustering in countries other than the Lao PDR [17-19]. One possible explanation for the household clustering is that household members share the same environmental risk factors including the proximity of housing location to breeding sites [20] and housing type [21]. Another possible explanation is that household members share the same risk behaviors such as not using a bed net $[9,20]$ and working in the forest [10]. The findings of the present study suggest the implementation of household-based strategies to achieve effective active case detection. A reactive case detection strategy that is linked to facility-based passive case detection can be an option for actively detecting cases in a setting where infections are highly clustered within households and the transmission intensity is low [22]. As reported elsewhere $[23,24]$, malaria patients who are identified through facility-based passive case detection can be used as an index case. Subsequently, individuals living with the index case are screened.

For such reactive case detection to be implemented in Xepon district or other similar settings in the Lao PDR, RDT would be used as a screening tool. Although the present study showed that RDT detected only $36.7 \%$ of the true positives confirmed by PCR in the study site and malaria elimination cannot be achieved by screening with the current generation of RDTs [25], the use of PCR for screening is not currently feasible in the rural districts of the Lao PDR. To increase the detection rate of secondary infections by screening with RDT, the following strategies should be incorporated. First, screening should focus on the households of index cases that do not possess a sufficient number of bed nets. In the present study, the households with multiple cases of infection were less likely to have a sufficient number of bed nets than were the households with a single case, although the difference was not statistically significant, possibly because of the small sample size $(n=36)$. Second, screening should focus on the households of index cases in which a household member has a history of fever/malaria. The present study found that two-week recall of a fever episode was reported only from the participants who had a RDT detectable infection. The present study also found that the RDT-positive participants were more likely to have reported a fever episode than were the RDT-negative participants (19.0 vs $7.9 \%$ ), although the difference was not significant. A previous study conducted in Savannakhet province reported that malaria-related symptoms in the past one year and a history of clinical malaria were predictive factors for the PCR-diagnosed malaria infections [9]. A Cambodian study [23] that assessed a reactive case detection approach using index cases found through passive case detection at a health facility or through a village malaria worker reported a significant association between the secondary infections detected by RDT and measured fever $\left(>37.2^{\circ} \mathrm{C}\right)$ and previous malaria. Finally, screening should focus on the households of index cases with an environmental risk factor. Another study conducted in the Lao PDR showed that the number of Anopheles mosquitoes that entered people's homes differed significantly according to the type of house, the presence of cattle, and the presence of wood smoke from cooking fires [21]. 
Table 8 Bivariate analysis of associations between malaria infection status determined by rapid diagnostic tests and predictive factors

\begin{tabular}{|c|c|c|c|c|c|c|}
\hline \multirow[t]{2}{*}{ Predictive factors } & \multicolumn{2}{|c|}{ Positive $(n=21)$} & \multicolumn{2}{|c|}{ Negative $(n=849)$} & \multirow[t]{2}{*}{ Positive rate (\%) } & \multirow[t]{2}{*}{$P$ value $^{a}$} \\
\hline & $\mathrm{n}$ & $\%$ & $\mathbf{n}$ & $\%$ & & \\
\hline \multicolumn{7}{|c|}{ Individual level factors } \\
\hline \multicolumn{7}{|c|}{ Age (years) } \\
\hline$<15$ & 14 & 66.7 & 445 & 52.4 & 3.1 & \multirow[t]{2}{*}{0.196} \\
\hline$\geq 15$ & 7 & 33.3 & 404 & 47.6 & 1.7 & \\
\hline \multicolumn{7}{|l|}{ Gender } \\
\hline Female & 11 & 52.4 & 459 & 54.1 & 2.3 & \multirow[t]{2}{*}{0.879} \\
\hline Male & 10 & 47.6 & 390 & 45.9 & 2.5 & \\
\hline \multicolumn{7}{|c|}{ Working forest at night } \\
\hline No & 20 & 95.2 & 817 & 96.2 & 2.4 & \multirow[t]{2}{*}{0.560} \\
\hline Yes & 1 & 4.8 & 32 & 3.8 & 3.0 & \\
\hline \multicolumn{7}{|c|}{ Fever episode in the last 2 weeks } \\
\hline No & 17 & 81.0 & 782 & 92.1 & 2.1 & \multirow[t]{2}{*}{0.084} \\
\hline Yes & 4 & 19.0 & 67 & 7.9 & 5.6 & \\
\hline \multicolumn{7}{|c|}{ Household level factors } \\
\hline \multicolumn{7}{|l|}{ Person-per-net ratio } \\
\hline$\leq 2.5$ & 8 & 38.1 & 412 & 48.3 & 1.9 & \multirow[t]{4}{*}{0.230} \\
\hline$>2.5$ and $<4.0$ & 9 & 42.9 & 227 & 26.7 & 3.8 & \\
\hline$\geq 4.0$ or no net & 2 & 9.5 & 154 & 18.1 & 1.3 & \\
\hline Unknown & 2 & 9.5 & 58 & 6.8 & 3.3 & \\
\hline \multicolumn{7}{|c|}{ Number of household members } \\
\hline$<5$ & 0 & 0.0 & 119 & 14.0 & 0.0 & \multirow[t]{3}{*}{0.171} \\
\hline $5-7$ & 11 & 52.4 & 376 & 44.3 & 2.8 & \\
\hline$\geq 8$ & 10 & 47.6 & 354 & 41.7 & 2.7 & \\
\hline \multicolumn{7}{|c|}{ Household with at least one additional infected member } \\
\hline No & 16 & 76.2 & 839 & 98.8 & 1.9 & \multirow[t]{2}{*}{$<0.001$} \\
\hline Yes & 5 & 23.8 & 10 & 1.2 & 33.3 & \\
\hline
\end{tabular}

${ }^{a}$ Chi square test or Fisher's exact test

The Cambodian study [23] showed, however, that the reactive case detection approach identified very few secondary infections. Among the members of the index cases, the positive rate of the secondary infections was $0.3 \%$ by RDT and $1.3 \%$ by PCR. The study recommended that the reactive case detection approach is not appropriate for very low transmission settings in which exposure to malaria occurs away from the community and there is a high level of treatment-seeking from the private sector. Given this recommendation, the reactive case detection approach could be appropriate in the study district of Xepon because one half of the infections occurred in children, who accounted for $52.8 \%$ of the participants, and a substantial proportion of the infections might have occurred within the village or on the fringe of the village. The possibility of such local transmission around the village can be supported by the findings of another study conducted in three villages in Xepon and Nong districts, Savannakhet province [10]. The study showed that many Anopheles dirus mosquitoes, which are a main vector in the Lao PDR, were captured in indoor light traps. Although treatment seeking from the private sector is also high in Xepon district, in which approximately $44 \%$ of the malaria cases were reported from the private sector in 2013/14, the private sector has been an integral part of the National Malaria Control Programme in the Lao PDR including Xepon district. Most importantly, there was a marked difference in the transmission intensity between the present study and the Cambodian study (positive rate by RDT: 2.4 vs $0.5 \%$ ).

A number of factors that were previously reported as risk factors from the Lao PDR and/or neighboring countries were not identified as risk factors in the present study site. Although not using a bed net was reported as a risk factor for malaria infection from Savannakhet province [9], almost all of the participants (99.2 \%) in the present study reported use of a bed net on the night before they were surveyed. Likewise, although sleeping 


\begin{tabular}{|c|c|c|c|}
\hline $\begin{array}{l}\text { Predictor vari- } \\
\text { ables }\end{array}$ & $\begin{array}{l}\text { Adjusted odds } \\
\text { ratio }^{\mathrm{a}}\end{array}$ & $\begin{array}{l}\text { Adjusted } 95 \% \\
\text { confidence } \\
\text { interval }\end{array}$ & $P$ value \\
\hline
\end{tabular}

\begin{tabular}{|c|c|c|c|}
\hline \multicolumn{4}{|c|}{ Individual level variables } \\
\hline \multicolumn{4}{|c|}{ Age (years) } \\
\hline$<15$ & 1.00 & Reference & \\
\hline$\geq 15$ & 0.52 & $0.18-1.46$ & 0.211 \\
\hline \multicolumn{4}{|l|}{ Gender } \\
\hline Female & 1.00 & Reference & \\
\hline Male & 1.01 & $0.38-2.69$ & 0.990 \\
\hline \multicolumn{4}{|c|}{ Working forest at midnight } \\
\hline No & 1.00 & Reference & \\
\hline Yes & 2.27 & $0.26-20.09$ & 0.461 \\
\hline \multicolumn{4}{|c|}{ Fever episode in the last 2 weeks } \\
\hline No & 1.00 & Reference & \\
\hline Yes & 2.54 & $0.73-8.87$ & 0.143 \\
\hline \multicolumn{4}{|c|}{ Household level variables } \\
\hline \multicolumn{4}{|c|}{ Person-per-net ratio } \\
\hline$\leq 2.5$ & 1.00 & Reference & \\
\hline$>2.5$ and $<4.0$ & 1.01 & $0.29-3.52$ & 0.986 \\
\hline$\geq 4.0$ or no net & 0.62 & $0.13-3.02$ & 0.558 \\
\hline \multicolumn{4}{|c|}{ Number of household members } \\
\hline$<8$ & 1.00 & Reference & \\
\hline$\geq 8$ & 0.38 & $0.10-1.42$ & 0.152 \\
\hline \multicolumn{4}{|c|}{ Household with at least one additional infected member } \\
\hline No & 1.00 & Reference & \\
\hline Yes & 56.69 & $9.38-342.61$ & $<0.001$ \\
\hline
\end{tabular}

adjusted for age, gender, working in forest, fever episode, person-per-net ratio, number of household members, and at least one additional infected member

away from home was reported as a risk factor for malaria infection from Attapeu province, Lao PDR [20], very few of the present participants $(0.9 \%)$ did this. Additionally, working in the forest at night, which is a known risk factor in Vietnam and Thailand [26, 27], was not common in the present study site. Because the present study failed to include more than one half of the population in the study site, it could possibly identify these previously reported risk factors if most of the population were included. For example, because the present study found that only $53.0 \%$ of the households owned a sufficient number of bed nets to fully cover all members, some of the non-participants might not have used a bed net.

A major limitation of this study is the low participation rate: only $47.8 \%$ of the population in the study villages participated. As Table 3 shows, compared to the participants, the non-participants were more likely to be male, children under the age of five, and adults (especially adult males). A possible reason for the lower participation rate among the children is that their guardians are unwilling to expose their young children to blood collection. A possible reason for the lower participation rate of the adults is that they worked outside the village during the period of data collection. Because of this limitation, the results of the present study could underestimate the risk of malaria infection among young children and adults while overestimating the risk among older children.

This study has two other limitations. First, because the data collection was confined to half of the population in the 10 villages from the HDSS area, the present sample was not representative of the population of Xepon district. Second, the survey took place between August and September (the rainy season) and, therefore, did not take seasonal malaria epidemiology into account or seasonal variation in the frequency of risk factors such as sleeping away from home, working in the forest at night, and not sleeping under a bed net. However, sleeping away from home is more frequently reported in the rainy season than in the dry season in the Lao PDR [15].

\section{Conclusions}

The present community-based study showed that $P$. falciparum infections were common but that infections involving $P$. vivax, which was rarely reported from the district healthcare facility, accounted for $21 \%$ of the malaria infections. This suggested that the burden of P. vivax infection is underestimated. Most (63\%) of the infections were asymptomatic and sub-microscopic and were highly clustered within households. To eliminate malaria in Xepon district and other similar settings in the Lao PDR, the National Malaria Control Programme should consider household-based control strategies. The findings of the present study suggest the use of a reactive case detection strategy targeting the household members of index cases.

\section{Additional files}

Additional file 1. Primer sequences.

Additional file 2. Characteristics of each participant with positive test results.

Additional file 3. Characteristics of each household with a positive participant.

\section{Abbreviations}

Lao PDR: The Lao People's Democratic Republic; RDT: rapid diagnostic test; ACT: artemisinin-based combination therapy; PCR: polymerase chain reaction; HDSS: health and demographic surveillance system; DNA: deoxyribonucleic acid.

\section{Authors' contributions}

TP and DN were the principal investigators and drafted the manuscript with the help of MI and SK. MI, PP, FN and JK contributed to data collection. MN contributed to the laboratory procedures and manuscript development. PP, 
JK, KM and TM contributed to the conception of the study. MI, BH, PTB, SK and TM contributed to the data analysis and reviewed the manuscript. All authors read and approved the final manuscript.

\section{Author details}

${ }^{1}$ Savannakhet Provincial Health Department, Phonsavangnuea Village, Kaysone-Phomvihan District, Savannakhet, Lao PDR. ${ }^{2}$ Department of Molecular and Cellular Parasitology, School of Medicine, Juntendo University, 2-1-1 Hongo, Bunkyo-ku, Tokyo 113-8421, Japan. ${ }^{3}$ SATREPS Project for Parasitic Diseases, Vientiane, Lao PDR. ${ }^{4}$ Department of Global Health, School of Health Sciences, University of the Ryukyus, 207 Uehara, Nishihara-cho, Okinawa 903-0215, Japan. ${ }^{5}$ Department of Tropical Medicine and Malaria, Research Institute, National Center for Global Health and Medicine, 1-21-1 Toyama, Shinjuku-ku, Tokyo 162-8655, Japan. ${ }^{6}$ Graduate School of Tropical Medicine and Global Health, Nagasaki University, 1-12-4 Sakamoto, Nagasaki-shi, Nagasaki 852-8523, Japan. ${ }^{7}$ Center of Malariology, Parasitology and Entomology, Ministry of Health, Vientiane, Lao PDR. ${ }^{8}$ Institut Pasteur du Laos, Sisattanak District, Vientiane, Lao PDR.

\section{Acknowledgements}

The authors sincerely thank the study participants and the field surveyors for their contributions to this study. The authors also thank the anonymous reviewers for their valuable inputs.

\section{Competing interests}

The authors declare that they have no competing interests.

\section{Availability of data and materials}

The data supporting the conclusions of this paper are included within the paper. Raw data may be obtained from the corresponding author upon request.

\section{Ethics approval and consent to participate}

This study was approved by the National Ethics Committee for Health Research, Ministry of Health, Lao PDR (No. 137/NECHR) and the Ethics Review Committee for Epidemiological Study, University of the Ryukyus, Japan (No. 176). The participants knew that their participation would be voluntary and that all data obtained would be confidential. Verbal and written consent was obtained from all of the participants before conducting the survey. The guardians of child participants consented to their participation.

\section{Funding}

This study was partly supported by a JICA/AMED SATREPS project for the "Development of innovative research technique in genetic epidemiology of malaria and other parasitic diseases in the Lao PDR for containing their expanding endemicity."

\section{Received: 17 May 2016 Accepted: 5 October 2016}

Published online: 18 October 2016

\section{References}

1. Lao Statistics Bureau. Vientiane capital: ministry of planning and investment, Lao PDR. 2016. http://www.Isb.gov.la/en/index.php. Accessed 18 Sept 2016.

2. Steering Committee for the Agricultural Census, Agricultural Census Office. Lao census of agriculture 2010/11 highlights. Vientiane: Agricultural Census Office; 2012.

3. Ministry of Health. National strategy for malaria control and pre-elimina tion 2011-2015. Vientiane: Ministry of Health; 2010.

4. WHO, UNICEF. The world malaria report 2015. Geneva: World Health Organization; 2015

5. Delacollette C, D'Souza C, Christophel E, Thimasarn K, Abdur R, Bell D, et al. Malaria trends and challenges in the Greater Mekong Subregion. Southeast Asian J Trop Med Public Health. 2009:40:674-91.

6. WHO. Malaria outbreaks remain worrisome but progress is being made in response and control. http://www.who.int/malaria/areas/greater mekong/lao-pdr-outbreaks-remain-worrisome/en/. 2014. Accessed 18 Sept 2016.
7. Nonaka D, Laimanivong S, Kobayashi J, Chindavonsa K, Kano S, Vanisaveth $\mathrm{V}$, et al. Is staying overnight in a farming hut a risk factor for malaria infection in a setting with insecticide-treated bed nets in rural Laos? Malar J. 2010;9:372.

8. Rattanaxay P, Phompida S, Kobayashi J. A review of malaria situation and its control in Lao PDR. In: Tongol-Rivera P, Kano S, editors. Asian parasitology. Vol 6, Malaria in Asia. Chiba: The Federation of Asian Parasitologists; 2005. p. 85-104.

9. Khaminsou N, Kritpetcharat O, Daduang J, Kritpetcharat P. A survey of malarial infection in endemic areas of Savannakhet province, Lao PDR and comparative diagnostic efficiencies of Giemsa staining, acridine orange staining, and semi-nested multiplex PCR. Parasitol Int. 2008:57:143-9.

10. Pongvongsa T, Ha H, Thanh L, Marchand RP, Nonaka D, Tojo B, et al. Joint malaria surveys lead towards improved cross-border cooperation between Savannakhet province, Laos and Quang Tri province, Vietnam. Malar J. 2012;11:262.

11. Nonaka D, Vongseththa K, Kobayashi J, Bounyadeth S, Kano S, Phompida $\mathrm{S}$, et al. Public and private sector treatment of malaria in Lao PDR. Acta Trop. 2009;112:283-7.

12. Ménard D, Khim N, Beghain J, Adegnika AA, Shafiul-Alam M, Amodu O, et al. A worldwide map of Plasmodium falciparum K13-propeller polymorphisms. N Engl J Med. 2016;374:2453-64.

13. WHO. Strategy for malaria elimination in the greater mekong subregion (2015-2030). Geneva: World Health Organization; 2015.

14. Nishimoto F, Kaneko S, Jiang H, Moji K. Impending demographic transitions in rural Laos: projections based on data from the health and demographic surveillance system. Jpn J Health Hum Ecol. 2014;80:54-9 (in Japanese)

15. Canier L, Khim N, Kim S, Sluydts V, Heng S, Dourng D, et al. An innovative tool for moving malaria PCR detection of parasite reservoir into the field. Malar J. 2013;12:405.

16. Nonaka D, Pongvongsa T, Nishimoto F, Nansounthavong P, Sato Y, Jiang $\mathrm{H}$, et al. Households with insufficient bednets in a village with sufficient bednets: Evaluation of household bednet coverage using bednet distribution index in Xepon district, Lao PDR. Trop Med Health. 2015:43:95-100.

17. Greenwood BM. The microepidemiology of malaria and its importance to malaria control. Trans R Soc Trop Med Hyg. 1989;83(Suppl):25-9.

18. Brooker S, Clarke S, Njagi JK, Polack S, Mugo B, Estambale B, et al. Spatial clustering of malaria and associated risk factors during an epidemic in a highland area of western Kenya. Trop Med Int Health. 2004;9:757-66.

19. Rulisa S, Kateera F, Bizimana JP, Agaba S, Dukuzumuremyi J, Baas L, et al. Malaria prevalence, spatial clustering and risk factors in a low endemic area of Eastern Rwanda: a cross sectional study. PLoS One. 2013;8:e69443.

20. Vythilingam I, Sidavong B, Chan ST, Phonemixay T, Vanisaveth V, Sisoulad $\mathrm{P}$, et al. Epidemiology of malaria in Attapeu Province, Lao PDR in relation to entomological parameters. Trans R Soc Trop Med Hyg. 2005;99:833-9.

21. Hiscox A, Khammanithong P, Kaul S, Sananikhom P, Luthi R, Hill N, et al. Risk factors for mosquito house entry in the Lao PDR. PLoS One. 2013;8:e62769.

22. Sturrock HJ, Hsiang MS, Cohen JM, Smith DL, Greenhouse B, Bousema $\mathrm{T}$, et al. Targeting asymptomatic malaria infections: active surveillance in control and elimination. PLoS Med. 2013;10:e1001467.

23. Hustedt J, Canavati SE, Rang C, Ashton RA, Khim N, Berne L, et al. Reactive case-detection of malaria in Pailin Province, Western Cambodia: lessons from a year-long evaluation in a pre-elimination setting. Malar J. 2016;15:132.

24. Littrell M, Sow GD, Ngom A, Ba M, Mboup BM, Dieye Y, et al. Case investigation and reactive case detection for malaria elimination in northern Senegal. Malar J. 2013;12:331.

25. von Seidlein $\mathrm{L}$. The failure of screening and treating as a malaria elimination strategy. PLoS Med. 2014;11:e1001595.

26. Erhart A, Ngo DT, Phan VK, Ta TT, Van Overmeir C, Speybroeck N, et al. Epidemiology of forest malaria in central Vietnam: a large scale crosssectional survey. Malar J. 2005:4:58.

27. Chaveepojnkamjorn W, Pichainarong N. Behavioral factors and malaria infection among the migrant population, Chiang Rai province. J Med Assoc Thai. 2005:88:1293-301. 